\title{
A NOTE ON BOUNDED SOLUTIONS OF AN ITERATIVE EQUATION
}

\author{
HOU YU ZHAO AND JIA LIU
}

Received 10 December, 2018

\begin{abstract}
In this paper, we use Schauder and Banach fixed point theorems to study the existence, uniqueness and stability of bounded nonhomogeneous iterative functional differential equations of the form

$$
x^{\prime}(t)=\lambda_{1} x(t)+\lambda_{2} x^{[2]}(t)+\ldots+\lambda_{n} x^{[n]}(t)+f(t) .
$$
\end{abstract}

2010 Mathematics Subject Classification: 39B12; 39B82

Keywords: iterative differential equation, bounded solutions, fixed point theorem

\section{INTRODUCTION}

Differential equations of the form

$$
x^{\prime}(t)=f\left(t, x(t), x\left(t-\tau_{1}(t)\right), \ldots, x\left(t-\tau_{k}(t)\right)\right)
$$

were discussed in [1] and [5]. In particularly, the delay functions $\tau_{j}(z), j=0,1, \ldots, k$ depend not only on unknown function, but also state, $\tau_{j}(z, x(z)), j=0,1, \ldots, k$ have been studied in many literatures. In [2], Cooke points out that it is highly desirable to establish the existence and stability of periodic solutions for equations of the form

$$
x^{\prime}(t)+a x(t-h(t, x(t)))=F(t),
$$

in which the lag $h(t, x(t))$ implicitly involves $x(t)$. Eder [3] considered the iterative functional differential equation

$$
x^{\prime}(t)=x^{[2]}(t)
$$

and obtains that every solution either vanishes identically or is strictly monotonic. Fečkan [4] studied the equation

$$
x^{\prime}(t)=f\left(x^{[2]}(t)\right)
$$

This work was partially supported by the National Natural Science Foundation of China (Grant No. 11501069), Science and Technology Research Program of Chongqing Municipal Education Commission (Grant No. KJQN201800502), Foundation of Youth Talent of Chongqing Normal University (Grant No. 02030307-00039). 
by obtaining an existence theorem for solutions satisfying $x(0)=0$. Staněk [9] studies the global properties of solutions of functional differential equation $x^{\prime}(t)=x(t)+$ $x(x(t))$, and shows that every solution either vanishes identically or is strictly monotonic. Later, in [6], $\mathrm{Si}, \mathrm{Li}$ and Cheng considered the equation

$$
x^{\prime}(t)=x^{[m]}(t)
$$

and establish sufficient conditions for the existence of analytic solutions. Si and Wang [7] discuss the smooth solutions of equation

$$
x^{\prime}(t)=\lambda_{1} x(t)+\lambda_{2} x^{[2]}(t)+\ldots+\lambda_{n} x^{[n]}(t)+f(t) .
$$

Particularly, in 2017, Zeng, Zhang, Lu and Zhang ([11]) considered a general $n$ dimensional mixed type differential equation with state dependence, give the existence of solutions without requiring the return condition, and apply the result to differential equations with iterates. For some various properties of solutions for several delay functional differential equations, we refer the interested reader to $[8,10]$.

In this paper, we consider the existence of bounded solutions of Eq. (1.1).

For $L>0$, define the set of all real valued continuous functions from $\mathbb{R}$ into $\mathbb{R}$

$$
\mathscr{B}(L)=\{x \in C(\mathbb{R}, \mathbb{R}):\|x\| \leq L, \forall t \in \mathbb{R}\} .
$$

Then $\mathcal{B}(L)$ is a Banach space with the norm

$$
\|x\|=\max _{t \in \mathbb{R}}|x(t)| .
$$

For $P>0, L \geq 0$, define the sets

$$
\mathscr{B}(L, P)=\left\{x \in \mathcal{P}_{T}:\|x\| \leq L,\left|x\left(t_{2}\right)-x\left(t_{1}\right)\right| \leq P\left|t_{2}-t_{1}\right|, \forall t_{1}, t_{2} \in \mathbb{R}\right\},
$$

which are closed convex and bounded subset of $\mathscr{B}(L)$, and we wish to find bounded functions $x \in \mathscr{B}(L, P)$ satisfying (1.1).

\section{EXISTENCE OF BOUNDED SOLUTIONS}

In this section, the existence of bounded solutions of Eq. (1.1) will be proved. Let us state the Schauder fixed point theorem, which will be used to prove our main theorem.

Theorem 1 (Schauder). Let $\Omega$ be a closed convex compact subset of a Banach space. Suppose that $A: \Omega \rightarrow \Omega$ is continuous. Then there exists $z \in \Omega$ with $z=A z$.

Throughout this paper, we assume that all functions are continuous with respect to their arguments and the following condition holds.

We begin with the following lemma. 
Lemma 1. For any $\varphi, \psi \in \mathscr{B}(L, P)$,

$$
\left\|\varphi^{[n]}-\psi^{[n]}\right\| \leq \sum_{j=0}^{n-1} P^{j}\|\varphi-\psi\|, n=1,2, \ldots .
$$

Proof. The result follows from the definition of $\mathscr{B}(L, P)$.

Now we rewrite (1.1) as a fixed point equation.

Lemma 2. Suppose $\lambda_{1}>0$. Then $x(t)$ is a solution of (1.1) if and only if

$$
x(t)=-\sum_{l=2}^{n} \lambda_{l} \int_{t}^{+\infty} x^{[l]}(s) e^{\lambda_{1}(t-s)} d s-\int_{t}^{+\infty} f(s) e^{\lambda_{1}(t-s)} d s .
$$

Proof. The proof is well-known but we present it here for the reader convenience. It is easy to see that Eq. (1.1) can be written in the form of

$$
x^{\prime}(t) e^{-\lambda_{1} t}-\lambda_{1} x(t) e^{-\lambda_{1} t}=\left(\sum_{l=2}^{n} \lambda_{l} x^{[l]}(t)+f(t)\right) e^{-\lambda_{1} t} .
$$

If $x(t)$ is a solution of (1.1), then integrating the above equality from $t$ to $+\infty$, we obtain

$$
x(t)=-\sum_{l=2}^{n} \lambda_{l} \int_{t}^{+\infty} x^{[l]}(s) e^{\lambda_{1}(t-s)} d s-\int_{t}^{+\infty} f(s) e^{\lambda_{1}(t-s)} d s .
$$

This completes the proof.

Lemma 3. Suppose $\lambda_{1}<0$. Then $x(t)$ is a solution of (1.1) if and only if

$$
x(t)=\sum_{l=2}^{n} \lambda_{l} \int_{-\infty}^{t} x^{[l]}(s) e^{\lambda_{1}(t-s)} d s+\int_{-\infty}^{t} f(s) e^{\lambda_{1}(t-s)} d s .
$$

Proof. As in Lemma 2, Eq. (1.1) can be written in the form of

$$
x^{\prime}(t) e^{-\lambda_{1} t}-\lambda_{1} x(t) e^{-\lambda_{1} t}=\left(\sum_{l=2}^{n} \lambda_{l} x^{[l]}(t)+f(t)\right) e^{-\lambda_{1} t} .
$$

If $x(t)$ is a solution of (1.1), then integrating the above equality from $-\infty$ to $t$, we obtain

$$
x(t)=\sum_{l=2}^{n} \lambda_{l} \int_{-\infty}^{t} x^{[l]}(s) e^{\lambda_{1}(t-s)} d s+\int_{-\infty}^{t} f(s) e^{\lambda_{1}(t-s)} d s .
$$

This completes the proof. 
Now we will need to construct a mapping satisfying the hypotheses of Theorem 1 . To this aim, we consider maps $A, A_{1}: \mathscr{B}(L, P) \rightarrow \mathbb{R}$ defined as follows:

$$
\begin{gathered}
(A x)(t)=-\sum_{l=2}^{n} \lambda_{l} \int_{t}^{+\infty} x^{[l]}(s) e^{\lambda_{1}(t-s)} d s-\int_{t}^{+\infty} f(s) e^{\lambda_{1}(t-s)} d s, \\
\left(A_{1} x\right)(t)=\sum_{l=2}^{n} \lambda_{l} \int_{-\infty}^{t} x^{[l]}(s) e^{\lambda_{1}(t-s)} d s+\int_{-\infty}^{t} f(s) e^{\lambda_{1}(t-s)} d s,
\end{gathered}
$$

Lemma 4. Operator $A$ and $A_{1}$ are Lipschitz continuous.

Proof. Take $x, y \in B(L, P), t \in \mathbb{R}$, then by (2.1) and (2.4), we have

$$
\begin{aligned}
|(A x)(t)-(A y)(t)| & \leq \sum_{l=2}^{n}\left|\lambda_{l}\right|\left|\int_{t}^{+\infty}\left(x^{[l]}(s)-y^{[l]}(s)\right) e^{\lambda_{1}(t-s)} d s\right| \\
& \leq \frac{1}{\lambda_{1}} \sum_{l=2}^{n} \sum_{j=0}^{l-1}\left|\lambda_{l}\right| P^{j}\|x-y\| .
\end{aligned}
$$

This proves $A$ is Lipschitz continuous.

Using the same method, it is easy to prove $A_{1}$ is Lipschitz continuous. This completes the proof.

It is easy to see by Arzela-Ascoli theorem that $\mathcal{B}(L, P)$ is compact. Now, we are ready to prove the following existence result.

Theorem 2. Suppose $f \in \mathscr{B}(\widetilde{L}, \widetilde{P}), \lambda_{1}>0, \sum_{l=2}^{n}\left|\lambda_{l}\right|<\min \left\{\lambda_{1}, \frac{1}{2}\right\}$ and the following inequalities hold

$$
\begin{aligned}
& \widetilde{L} \leq\left(\lambda_{1}-\sum_{l=2}^{n}\left|\lambda_{l}\right|\right) L, \\
& \widetilde{P} \leq\left(\frac{1}{2}-\sum_{l=2}^{n}\left|\lambda_{l}\right|\right) P,
\end{aligned}
$$

then Eq. (1.1) has a solution in $\mathscr{B}(L, P)$.

Proof. First, for any $x, y \in \mathscr{B}(L, P)$, by (2.6), we have

$$
\begin{aligned}
|(A x)(t)| & \leq \sum_{l=2}^{n}\left|\lambda_{l}\right|\left|\int_{t}^{+\infty} x^{[l]}(s) e^{\lambda_{1}(t-s)} d s\right|+\left|\int_{t}^{+\infty} f(s) e^{\lambda_{1}(t-s)} d s\right| \\
& \leq \frac{L}{\lambda_{1}} \sum_{l=2}^{n}\left|\lambda_{l}\right|+\frac{\widetilde{L}}{\lambda_{1}}
\end{aligned}
$$




$$
\leq L
$$

Next, assuming $t_{1} \leq t_{2}$, by (2.7), we obtain

$$
\begin{aligned}
& \left|\int_{t_{2}}^{+\infty} x^{[l]}(s) e^{\lambda_{1}\left(t_{2}-s\right)} d s-\int_{t_{1}}^{+\infty} x^{[l]}(s) e^{\lambda_{1}\left(t_{1}-s\right)} d s\right| \\
& \leq\left|\int_{t_{2}}^{+\infty} x^{[l]}(s)\left(e^{\lambda_{1} t_{2}}-e^{\lambda_{1} t_{1}}\right) e^{-\lambda_{1} s} d s\right|+\left|\int_{t_{1}}^{t_{2}} x^{[l]}(s) e^{\lambda_{1}\left(t_{1}-s\right)} d s\right| \\
& \leq \frac{P}{\lambda_{1}}\left(1-e^{-\lambda_{1}\left(t_{2}-t_{1}\right)}\right)+\frac{P}{\lambda_{1}}\left(1-e^{-\lambda_{1}\left(t_{2}-t_{1}\right)}\right) \\
& \leq \frac{2 P}{\lambda_{1}} e^{\xi}\left(t_{2}-t_{1}\right) \lambda_{1} \\
& \leq 2 P\left(t_{2}-t_{1}\right)
\end{aligned}
$$

and

$$
\begin{aligned}
& \left|\int_{t_{2}}^{+\infty} f(s) e^{\lambda_{1}\left(t_{2}-s\right)} d s-\int_{t_{1}}^{+\infty} f(s) e^{\lambda_{1}\left(t_{1}-s\right)} d s\right| \\
& \leq\left|\int_{t_{2}}^{+\infty} f(s)\left(e^{\lambda_{1} t_{2}}-e^{\lambda_{1} t_{1}}\right) e^{-\lambda_{1} s} d s\right|+\left|\int_{t_{1}}^{t_{2}} f(s) e^{\lambda_{1}\left(t_{1}-s\right)} d s\right| \\
& \leq \frac{2 \widetilde{P}}{\lambda_{1}} e^{\xi}\left(t_{2}-t_{1}\right) \lambda_{1} \\
& \leq 2 \widetilde{P}\left(t_{2}-t_{1}\right),
\end{aligned}
$$

where $-\lambda_{1}\left(t_{2}-t_{1}\right)<\xi<0$,

By (2.8), (2.9) and (2.7), we have

$$
\begin{aligned}
& \left|(A x)\left(t_{2}\right)-(A x)\left(t_{1}\right)\right| \\
& \leq \sum_{l=2}^{n}\left|\lambda_{l}\right|\left|\int_{t_{2}}^{+\infty} x^{[l]}(s) e^{\lambda_{1}\left(t_{2}-s\right)} d s-\int_{t_{1}}^{+\infty} x^{[l]}(s) e^{\lambda_{1}\left(t_{1}-s\right)} d s\right| \\
& \quad+\left|\int_{t_{2}}^{+\infty} f(s) e^{\lambda_{1}\left(t_{2}-s\right)} d s-\int_{t_{1}}^{+\infty} f(s) e^{\lambda_{1}\left(t_{1}-s\right)} d s\right| \\
& \leq 2\left(\sum_{l=2}^{n}\left|\lambda_{l}\right| P+\widetilde{P}\right)\left(t_{2}-t_{1}\right) \\
& \leq P\left(t_{2}-t_{1}\right) .
\end{aligned}
$$


So by Lemma 4, we see that all conditions of Schauder's theorem are satisfied on $\mathscr{B}(L, P)$. Thus there exists a fixed point $x$ in $\mathcal{B}(L, P)$ such that $x=A x$, from Lemma 2, $x$ is a bounded solution of Eq. (1.1). This completes the proof.

Theorem 3. Suppose $f \in \mathscr{B}(\widetilde{L}, \widetilde{P}), \lambda_{1}<0, \sum_{l=2}^{n}\left|\lambda_{l}\right|<\min \left\{-\lambda_{1}, \frac{1}{2}\right\}$,

$$
\widetilde{L} \leq\left(-\lambda_{1}-\sum_{l=2}^{n}\left|\lambda_{l}\right|\right) L,
$$

and (2.7) holds, then Eq. (1.1) has a solution in $\mathscr{B}(L, P)$.

Proof. First, for any $x, y \in \mathscr{B}(L, P)$, by (2.10), we have

$$
\begin{aligned}
\left|\left(A_{1} x\right)(t)\right| & \leq \sum_{l=2}^{n}\left|\lambda_{l}\right|\left|\int_{-\infty}^{t} x^{[l]}(s) e^{\lambda_{1}(t-s)} d s\right|+\left|\int_{-\infty}^{t} f(s) e^{\lambda_{1}(t-s)} d s\right| \\
& \leq-\frac{L}{\lambda_{1}} \sum_{l=2}^{n}\left|\lambda_{l}\right|-\frac{\widetilde{L}}{\lambda_{1}} \\
& \leq L .
\end{aligned}
$$

Next, assuming $t_{1} \leq t_{2}$, we obtain

$$
\begin{aligned}
& \left|\int_{-\infty}^{t_{2}} x^{[l]}(s) e^{\lambda_{1}\left(t_{2}-s\right)} d s-\int_{-\infty}^{t_{1}} x^{[l]}(s) e^{\lambda_{1}\left(t_{1}-s\right)} d s\right| \\
& \leq\left|\int_{-\infty}^{t_{1}} x^{[l]}(s)\left(e^{\lambda_{1} t_{2}}-e^{\lambda_{1} t_{1}}\right) e^{-\lambda_{1} s} d s\right|+\left|\int_{t_{1}}^{t_{2}} x^{[l]}(s) e^{\lambda_{1}\left(t_{2}-s\right)} d s\right| \\
& \leq-\frac{P}{\lambda_{1}}\left(1-e^{\lambda_{1}\left(t_{2}-t_{1}\right)}\right)-\frac{P}{\lambda_{1}}\left(1-e^{\lambda_{1}\left(t_{2}-t_{1}\right)}\right) \\
& \leq \frac{2 P}{-\lambda_{1}} e^{\xi_{1}}\left(t_{2}-t_{1}\right)\left(-\lambda_{1}\right) \\
& \leq 2 P\left(t_{2}-t_{1}\right)
\end{aligned}
$$

and

$$
\begin{aligned}
& \left|\int_{-\infty}^{t_{2}} f(s) e^{\lambda_{1}\left(t_{2}-s\right)} d s-\int_{-\infty}^{t_{1}} f(s) e^{\lambda_{1}\left(t_{1}-s\right)} d s\right| \\
& \leq\left|\int_{-\infty}^{t_{1}} f(s)\left(e^{\lambda_{1} t_{2}}-e^{\lambda_{1} t_{1}}\right) e^{-\lambda_{1} s} d s\right|+\left|\int_{t_{1}}^{t_{2}} f(s) e^{\lambda_{1}\left(t_{2}-s\right)} d s\right| \\
& \leq \frac{2 \widetilde{P}}{-\lambda_{1}} e^{\xi_{1}}\left(t_{2}-t_{1}\right)\left(-\lambda_{1}\right) \\
& \leq 2 \widetilde{P}\left(t_{2}-t_{1}\right)
\end{aligned}
$$


where $\lambda_{1}\left(t_{2}-t_{1}\right)<\xi_{1}<0$.

By (2.11), (2.12) and (2.10), we have

$$
\begin{aligned}
& \left|(A x)\left(t_{2}\right)-(A x)\left(t_{1}\right)\right| \\
& \leq \sum_{l=2}^{n}\left|\lambda_{l}\right|\left|\int_{-\infty}^{t_{2}} x^{[l]}(s) e^{\lambda_{1}\left(t_{2}-s\right)} d s-\int_{-\infty}^{t_{1}} x^{[l]}(s) e^{\lambda_{1}\left(t_{1}-s\right)} d s\right| \\
& \quad+\left|\int_{-\infty}^{t_{2}} f(s) e^{\lambda_{1}\left(t_{2}-s\right)} d s-\int_{-\infty}^{t_{1}} f(s) e^{\lambda_{1}\left(t_{1}-s\right)} d s\right| \\
& \leq 2\left(\sum_{l=2}^{n}\left|\lambda_{l}\right| P+\widetilde{P}\right)\left(t_{2}-t_{1}\right) \\
& \leq P\left(t_{2}-t_{1}\right) .
\end{aligned}
$$

So by Lemma 4, we see that all conditions of Schauder's theorem are satisfied on $\mathcal{B}(L, P)$. Thus there exists a fixed point $x$ in $\mathcal{B}(L, P)$ such that $x=A x$, from Lemma 3, $x$ is a bounded solution of Eq. (1.1). This completes the proof.

From Theorems 2 and 3 we have the following theorem.

Theorem 4. Suppose $f \in \mathscr{B}(\widetilde{L}, \widetilde{P}), \lambda_{1} \neq 0, \sum_{l=2}^{n}\left|\lambda_{l}\right|<\min \left\{\left|\lambda_{1}\right|, \frac{1}{2}\right\}$ and the following inequalities hold

$$
\begin{aligned}
& \widetilde{L} \leq\left(\left|\lambda_{1}\right|-\sum_{l=2}^{n}\left|\lambda_{l}\right|\right) L, \\
& \widetilde{P} \leq\left(\frac{1}{2}-\sum_{l=2}^{n}\left|\lambda_{l}\right|\right) P,
\end{aligned}
$$

then Eq. (1.1) has a solution in $\mathcal{B}(L, P)$.

\section{UNIQUENESS AND STABILITY}

In this section, uniqueness and stability of (1.1) will be proved.

Theorem 5. In addition to the assumption of Theorem 4, suppose that

$$
\Gamma=\frac{1}{\left|\lambda_{1}\right|} \sum_{l=2}^{n} \sum_{j=0}^{l-1}\left|\lambda_{l}\right| P^{j}<1,
$$

then Eq. (1.1) has a unique solution in $\mathcal{B}(L, P)$.

Proof. We know from the proof of Theorem 2 that $A: \mathcal{B}(L, P) \rightarrow \mathcal{B}(L, P)$. Moreover, by Lemma 2, we get

$$
\|A \varphi-A \psi\| \leq \Gamma\|\varphi-\psi\|, \quad \varphi, \psi \in \mathscr{B}(L, P)
$$


(3.1) means $\Gamma<1$, so the fixed point must be unique by the Banach fixed point theorem.

Theorem 6. The unique solution obtained in Theorem 5 depends continuously on the given constants $\lambda_{l}$ and function $f(t)$, for $l=1, \ldots, n$.

Proof. First, assuming $\lambda_{1}, \mu_{1}>0$. Let constants $\lambda_{l}, \mu_{l}, l=2, \ldots, n$ and functions $f(t), \widetilde{f}(t) \in \mathscr{B}(\widetilde{L}, \widetilde{P})$ be given. Then we consider the corresponding operators $A, \widetilde{A}$, defined by (2.4), respectively. Assuming corresponding conditions (2.6), (2.7) and (3.1), there are two unique corresponding functions $x(t)$ and $\widetilde{x}(t)$ in $\mathcal{B}(L, P)$ such that

$$
x=A x, \quad \widetilde{x}=\widetilde{A} \widetilde{x} .
$$

Then we have

$$
\|x-\widetilde{x}\| \leq\|A x-A \widetilde{x}\|+\|A \widetilde{x}-\widetilde{A} \widetilde{x}\| \leq \Gamma\|x-\widetilde{x}\|+\|A \widetilde{x}-\widetilde{A} \widetilde{x}\|,
$$

which implies

$$
\|x-\widetilde{x}\| \leq \frac{\|A \widetilde{x}-\widetilde{A} \widetilde{x}\|}{1-\Gamma} .
$$

If $\lambda_{1} \geq \mu_{1}>0$, then

$$
\begin{aligned}
& \int_{t}^{+\infty}\left|e^{\lambda_{1}(t-s)}-e^{\mu_{1}(t-s)}\right| d s \\
& =\int_{t}^{+\infty}\left(e^{\mu_{1}(t-s)}-e^{\lambda_{1}(t-s)}\right) d s=\frac{1}{\mu_{1}}-\frac{1}{\lambda_{1}}
\end{aligned}
$$

If $\mu_{1} \geq \lambda_{1}>0$, then

$$
\begin{aligned}
& \int_{t}^{+\infty}\left|e^{\lambda_{1}(t-s)}-e^{\mu_{1}(t-s)}\right| d s \\
& =\int_{t}^{+\infty}\left(e^{\lambda_{1}(t-s)}-e^{\mu_{1}(t-s)}\right) d s=\frac{1}{\lambda_{1}}-\frac{1}{\mu_{1}}
\end{aligned}
$$

From (3.3) and (3.4), we have

$$
\begin{aligned}
& |(A \widetilde{x})(t)-(\widetilde{A} \widetilde{x})(t)| \\
& \leq \sum_{l=2}^{n}\left|\lambda_{l}-\mu_{l}\right|\left|\int_{t}^{+\infty} \widetilde{x}^{[l]}(s) e^{\lambda_{1}(t-s)} d s\right| \\
& \quad+\sum_{l=2}^{n}\left|\mu_{l}\right| \int_{t}^{+\infty}\left|\widetilde{x}^{[l]}(s)\right|\left|e^{\lambda_{1}(t-s)}-e^{\mu_{1}(t-s)}\right| d s \\
& \quad+\int_{t}^{+\infty}|f(s)-\widetilde{f}(s)| e^{\lambda_{1}(t-s)} d s+\int_{t}^{+\infty}|\widetilde{f}(s)|\left|e^{\lambda_{1}(t-s)}-e^{\mu_{1}(t-s)}\right| d s
\end{aligned}
$$




$$
\begin{aligned}
& \leq \frac{P}{\lambda_{1}} \sum_{l=2}^{n}\left|\lambda_{l}-\mu_{l}\right|+P \sum_{l=2}^{n}\left|\mu_{l}\right|\left|\frac{1}{\lambda_{1}}-\frac{1}{\mu_{1}}\right|+\frac{1}{\lambda_{1}}\|f-\widetilde{f}\|+P\left|\frac{1}{\lambda_{1}}-\frac{1}{\mu_{1}}\right| \\
& =\left(P+P \sum_{l=2}^{n}\left|\mu_{l}\right|\right)\left|\frac{1}{\lambda_{1}}-\frac{1}{\mu_{1}}\right|+\frac{P}{\lambda_{1}} \sum_{l=2}^{n}\left|\lambda_{l}-\mu_{l}\right|+\frac{1}{\lambda_{1}}\|f-\widetilde{f}\|,
\end{aligned}
$$

by (3.2), $\|x-\widetilde{x}\|$ tends to 0 when $\mu_{l}$ tends to $\lambda_{l}$ and $\widetilde{f}$ tends to $f$.

For the case $\lambda_{1}, \mu_{1}<0$, we have the similar proof. This completes the proof.

\section{EXAMPLES}

First, we show that the conditions in Theorem 2 do not self-contradict. Consider the following equation:

\section{Example 1.}

$$
x^{\prime}(t)=\frac{1}{3} x(t)+\frac{1}{4} x^{[2]}(t)+\frac{1}{12} \sin t
$$

where $\lambda_{1}=\frac{1}{3}, \lambda_{2}=\frac{1}{4}, f(t)=\frac{1}{12} \sin t$. Take $P=1, L=1, \widetilde{P}=\frac{1}{12}, \widetilde{L}=\frac{1}{12}$. A simple calculation yields

$$
\begin{aligned}
& \widetilde{L}=\frac{1}{12} \leq \frac{1}{12}=\left(\lambda_{1}-\sum_{l=2}^{n}\left|\lambda_{l}\right|\right) L, \\
& \widetilde{P}=\frac{1}{12} \leq \frac{1}{4}=\left(\frac{1}{2}-\sum_{l=2}^{n}\left|\lambda_{l}\right|\right) P .
\end{aligned}
$$

By Theorem 4, Eq. (4.1) has a bounded solution $x$ such that $|x(t)| \leq 1$, and $\mid x\left(t_{2}\right)-$ $x\left(t_{1}\right)|\leq| t_{2}-t_{1} \mid, \forall t_{1}, t_{2} \in \mathbb{R}$.

Next, we apply Theorem 3 .

Example 2. Consider equation:

$$
x^{\prime}(t)=\frac{1}{3} x(t)+\frac{1}{4} x^{[2]}(t)+\frac{1}{16} \sin t,
$$

where $\lambda_{1}=\frac{1}{3}, \lambda_{2}=\frac{1}{4}, f(t)=\frac{1}{12} \sin t$. Take $P=\frac{7}{24}, L=1, \widetilde{P}=\frac{1}{16}, \widetilde{L}=\frac{1}{16}$. A simple calculation yields

$$
\begin{aligned}
& \widetilde{L}=\frac{1}{16} \leq \frac{1}{12}=\left(-\lambda_{1}-\sum_{l=2}^{n}\left|\lambda_{l}\right|\right) L, \\
& \widetilde{P}=\frac{1}{16} \leq \frac{7}{96}=\left(\frac{1}{2}-\sum_{l=2}^{n}\left|\lambda_{l}\right|\right) P .
\end{aligned}
$$


By Theorem 2, Eq. (4.1) has a bounded solution $x$ such that $|x(t)| \leq 1$, and $\mid x\left(t_{2}\right)-$ $x\left(t_{1}\right)|\leq| t_{2}-t_{1} \mid, \forall t_{1}, t_{2} \in \mathbb{R}$. Furthermore,

$$
\Gamma=\frac{1}{\left|\lambda_{1}\right|} \sum_{l=2}^{n} \sum_{j=0}^{l-1}\left|\lambda_{l}\right| P^{j}=\frac{31}{32}<1,
$$

by Theorem 5, we know this bounded solution is unique.

Example 3.

$$
x^{\prime}(t)=\frac{1}{3} x(t)+\frac{1}{4} x^{[2]}(t)+\delta \sin t
$$

where $\delta>0$ is a parameter. As Example 1 or 2 , here $\lambda_{1}=\frac{1}{3}, \lambda_{2}=\frac{1}{4}, f(t)=\delta \sin t$ and $\widetilde{P}=\delta, \widetilde{L}=\delta$. Next, we consider $L(\delta)$ and $P(\delta)$ as variables to be defined by $\delta$. Then (2.6) and (2.7) have the forms

$$
\begin{gathered}
\delta \leq \frac{1}{12} L(\delta), \\
\delta \leq \frac{1}{4} P(\delta), \\
\frac{3}{4}(1+P(\delta))<1,
\end{gathered}
$$

First, by (4.4) and (4.5) we have

$$
L(\delta) \geq 12 \delta
$$

and

$$
P(\delta) \geq 4 \delta
$$

Next, (4.6) shows us

$$
P(\delta)<\frac{1}{3}
$$

By (4.8) and (4.9), we have

$$
4 \delta \leq P(\delta)<\frac{1}{3}
$$

and

$$
0<\delta \leq \frac{1}{12} \doteq 0.083333 .
$$

Thus, if we taking $P(\delta)$ and $L(\delta)$ satisfy (4.7) and (4.8), Theorem 2 are satisfied and Eq. (4.3) has a $2 \pi$-periodic solution such that $|x(t)| \leq P(\delta)$ and $\left|x\left(t_{2}\right)-x\left(t_{1}\right)\right| \leq$ $L(\delta)\left|t_{2}-t_{1}\right|, \forall t_{1}, t_{2} \in \mathbb{R}$. Furthermore, if we taking $L(\delta)$ and $P(\delta)$ satisfy (4.7)(4.11), by Theorem 5 , we know the $2 \pi$-periodic solution of (4.3) is a unique one. 
Remark 1. Obviously, Example 1 satisfies conditions (4.7) and (4.8), Example 2 satisfies conditions (4.7)-(4.11).

\section{ACKNOWLEDGEMENT}

The authors are grateful to the Professor Weinian Zhang for his helpful comments and suggestions.

\section{REFERENCES}

[1] R. Bellman and K.L. Cooke, Differential-Difference Equations. New York: Acadmic Press, 1963.

[2] K. Cooke, Functional differential systems: some models and perturbation problems, in: Proceedings of the International Symposium on Differential Equations and Dynamical Systems. New York: Acadmic Press, 1967.

[3] E. Eder, "The functional differential equation $x^{\prime}(t)=x(x(t))$." J. Differential Equations., vol. 54, pp. 390-400, 1984, doi: 10.1016/0022-0396(84)90150-5.

[4] M. Fečkan, "On a certain type of functional differential equations." Math. Slovaca., vol. 43, pp. 39-43, 1993.

[5] J. Hale, Theory of Functional Differential Equations. New York: Acadmic Press, 1977.

[6] J.G. Si, W.R. Li, and S.S. Cheng, "Analytic solutions of an iterative functional differential equation." Comput. Math. Appl., vol. 33, pp. 47-51, 1997, doi: 10.1016/S0898-1221(97)00030-8.

[7] J.G. Si and X.P. Wang, "Smooth solutions of a nonhomogeneous iterative functional differential equation with variable coefficients." J. Math. Anal. Appl., vol. 226, pp. 377-392, 1998, doi: 10.1006/jmaa.1998.6086.

[8] S.N. Chow, "Existence of periodic solutions of autonomous functional differential equations." $J$. Differential Equations., vol. 15, pp. 350-378, 1974, doi: 10.1016/0022-0396(74)90084-9.

[9] S. Staněk, " On global properties of solutions of functional differential equation $x^{\prime}(t)=x(t)+$ $x(x(t)) . ”$ Dynam. Systems Appl., vol. 4, pp. 263-278, 1995.

[10] T.A. Burton, Stability by Fixed Point Theory for Functional Differential Equations. New York: Dover, 2006.

[11] Y.Y. Zeng, P.P. Zhang, T.T. Lu, and W.N. Zhang, "Existence of solutions for a mixed type differential equation with state-dependence." J. Math. Anal. Appl., vol. 453, pp. 629-644, 2017, doi: 10.1016/j.jmaa.2017.04.020.

Authors' addresses

Hou Yu Zhao

Chongqing Normal University, School of Mathematics, Chongqing, 401331, P.R.China

E-mail address: houyu19@gmail.com

Jia Liu

Shandong Urban Construction Vocational College,, Department of Architectural Economic Management, Jinan, Shandong 250103, P.R.China

E-mail address: $22151241 @ q q . \mathrm{com}$ 